Published in final edited form as:

Environ Res. 2020 November ; 190: 109994. doi:10.1016/j.envres.2020.109994.

\title{
Exposure to Environmental Chemical Mixtures is Associated with Nasal Colonization by Staphylococcus aureus: NHANES 2001-2004
}

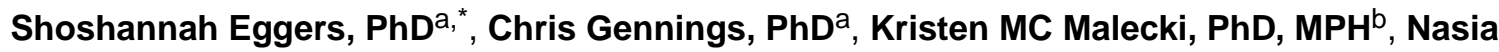 \\ Safdar, MD, PhD ${ }^{\mathrm{c}, \mathrm{d}}$, Manish Arora, PhD, MPH, BDS, FICDa \\ a. Department of Environmental Medicine and Public Health, Icahn School of Medicine at Mount \\ Sinai, 1 Gustave L. Levy Place, Box 1057, New York, NY 10029, USA; \\ b. Department of Population Health Sciences, School of Medicine and Public Health, University of \\ Wisconsin - Madison, 610 Walnut St, WARF 707, Madison, WI 53726, USA; \\ c. Division of Infectious Disease, Department of Medicine, School of Medicine and Public Health, \\ University of Wisconsin - Madison, UW Med Fndtn Centennial Bldg, 1685 Highland Ave, Madison, \\ WI 53705, USA;
}

d. William S. Middleton Veterans Affairs Medical Center, 2500 Overlook Terrace, Madison, WI 53705, USA

\section{Abstract}

Background: Understanding the health effects of exposure to chemical mixtures is critically important given the broad range of concurrent exposures throughout the life-course. While investigations of environmental chemicals and components of the human microbiome are becoming more common, few have examined associations with chemical mixtures. This study assesses the association between exposure to mixtures of 66 different environmental chemicals and nasal colonization of Staphylococcus aureus (SA) and methicillin resistant SA (MRSA).

Methods: Data came from the National Health and Nutrition Examination Survey (NHANES) 2001-2004. The analytical sample consists of 10312 participants, age 6 years and older, subdivided into 8 groups with different chemical exposure mixtures. Within each of 6 chemical classes (metals, phthalates, polycyclic aromatic hydrocarbons (PAHs), polybrominated diphenyl ethers (PBDEs), polyfluorochemicals (PFCs), and phenols), weighted quantile sum (WQS)

*Corresponding Author: shoshannah.eggers@mssm.edu.

Shoshannah Eggers: Conceptualization, Methodology, Formal analysis, Writing - Original Draft, Review \& Editing. Chris Gennings: Methodology, Writing - Review \& Editing. Kristen MC Malecki: Conceptualization, Writing - Review \& Editing. Nasia Safdar: Conceptualization, Writing - Review \& Editing. Manish Arora: Conceptualization, Methodology, Writing - Review \& Editing, Supervision.

Publisher's Disclaimer: This is a PDF file of an unedited manuscript that has been accepted for publication. As a service to our customers we are providing this early version of the manuscript. The manuscript will undergo copyediting, typesetting, and review of the resulting proof before it is published in its final form. Please note that during the production process errors may be discovered which could affect the content, and all legal disclaimers that apply to the journal pertain.

Declaration of interests

ख The authors declare that they have no known competing financial interests or personal relationships that could have appeared to influence the work reported in this paper. 
regression was used to analyze the joint association of the component compounds and nasal SA colonization. WQS was also used to assess the joint association of 3 chemical mixtures (metals, metal and PAHs, and metal and triclosan) and nasal MRSA colonization. All regression models were adjusted for confounders.

Results: The analytical sample was between ages $6-85$, slightly more female, and predominantly non-smokers. Prevalence of SA carriage was $29.2 \%$, and MRSA colonization prevalence was $1.2 \%$. Within each chemical class, odds of SA colonization increased statistically significantly with exposure to mixtures of metals $(\mathrm{OR}=1.11,95 \% \mathrm{CI}=1.02-1.20)$, phthalates $(\mathrm{OR}=1.09$, $95 \% \mathrm{CI}=1.04-1.14)$, and phenols $(\mathrm{OR}=1.08,95 \% \mathrm{CI}=1.01-1.15)$. Exposure to a mixture of metals combined with PAHs was also associated with increased odds of MRSA carriage $(\mathrm{OR}=1.38,95 \% \mathrm{CI}=1.02-1.86)$.

Conclusion: Results indicate an association between multiple environmental chemical mixtures and SA colonization, including MRSA. These findings support the need for further analysis of associations between chemical mixtures and SA colonization, as well as other components of the human microbiome.

\section{Keywords}

Environmental exposure; Co-exposure; Chemical mixture; MRSA; Staphylococcus aureus; Antibiotic resistance

\section{INTRODUCTION}

Given that people are concurrently exposed to many environmental chemicals through the life-course (Bopp et al., 2018), investigating the human health effects of exposure to chemical mixtures is a recognized research priority by the National Institutes of Health (Carlin Danielle J. et al., 2013). Chemical-microbial interactions may also play an important role in the human exposome and have implications for host susceptibility and immune response, however, few population-based studies have examined the effects of chemical mixtures on the composition of the human microbiome. Studies have shown individual effects of environmental chemicals on components of the microbiome, including altered composition and function, in many animal trials (Ba et al., 2017; Chi et al., 2017; Gao et al., 2017; Hu et al., 2016; Lai et al., 2016) and some epidemiologic studies (Bisanz et al., 2014; Eggers et al., 2019, 2018; Zhai et al., 2019). It stands to reason that mixtures of these chemicals may have either additive or interactive effects.

Staphylococcus aureus (SA) is a bacterial component of the human microbiome, found on the skin and mucosal surfaces, and colonizes approximately $30 \%$ of the US population (Graham et al., 2006; Human Microbiome Project Consortium, 2012). While typically commensal, SA can also cause infection leading to significant morbidity and mortality, particularly infections of methicillin resistant Staphylococcus aureus (MRSA). Although the nasal microbiome varies between individuals, Corynebacterium, Propionibacterium, and Staphylococcus are the most abundant genera (Frank et al., 2010; Human Microbiome Project Consortium, 2012). Factors associated with colonization by SA include other infections, rheumatoid arthritis, smoking, obesity and hospital employment (Sakr et al., 
2018). However, few studies have investigated associations with environmental factors. Our previous analysis showed associations between blood lead and cadmium levels and nasal colonization by SA in the National Health and Nutrition Examination Study (NHANES) (Eggers et al., 2018), however, we did not consider co-exposure.

While metals have been among the most investigated environmental exposures in association with the mammalian microbiome and antibiotic resistant bacteria, investigation of the effects of exposures from other chemical classes are more limited. Some sparse evidence has shown exposure to individual PAHs, phthalates, and phenols to be associated with bacterial toxicity, shifts in microbiome composition and diversity, and selection for antibiotic resistance in some cases (Hu et al., 2016; Iszatt et al., 2019; Lai et al., 2016; Tóth et al., 2016; Wang et al., 2017). More evidence of associations between many different classes of environmental chemicals and components of the human microbiome are needed, including analysis of coexposures within and across chemical classes.

The aim of this study is to examine the association between mixtures of environmental chemicals with nasal carriage of SA, as an indicator of association with the human microbiome. We also examined associations between environmental chemical mixtures and nasal MRSA colonization. We hypothesized that chemical mixtures would be associated with SA carriage. We analyzed publicly available NHANES data from 2001-2004, when screening for SA and MRSA occurred. Analyses were performed using weighted quantile sum (WQS) regression which supports the use of multiple highly correlated exposures, and can identify the most highly influential chemical within the mixture (Carrico et al., 2015). We analyzed the association of 6 chemical mixtures with SA colonization, and 3 chemical mixtures with MRSA colonization.

\section{METHODS}

\subsection{Data Source}

Data for this analysis come from NHANES. In 1999 NHANES began operating as a continuous, annual survey, representative of the noninstitutionalized civilian United States population, with approximately 5000 participants per year (Zipf et al., 2013). It consists of in-person interviews and physical examinations, providing survey data for national estimates for many health related determinants and outcomes. During the physical exams, biological specimens are collected, which are then tested for a range of analytes, including many environmental exposure biomarkers and health outcome markers. The continuous nature of the survey allowed for individual tests and components to change as needed. The outcome of interest for this analysis was nasal SA, which was assessed as the need emerged from 20012004. Therefore, this analysis is limited to data collected in 2001-2004. The analytical sample for each statistical model was based on data availability for all variables in each model. Due to the small amount of missing SA and chemical exposure data, missing data was treated as missing at random. This study is exempt from review by the Mount Sinai IRB as the data are de-identified and publicly available. 


\subsection{Variables}

2.2.1. Exposures-With our interest in using a chemical mixtures approach in this analysis, we considered many classes of chemical exposures, however, due to NHANES's sampling procedure, data overlap among exposures was limited. All NHANES data and analytical procedures can be found at https://wwwn.cdc.gov/nchs/nhanes/default.aspx. We chose 66 exposure analytes based on our interest in environmental chemical exposures and data availability. We grouped these exposures into eight chemical mixture groups, six within-chemical-class mixtures, and two across-chemical-class mixtures, with varying sample sizes, as shown in Table 1 .

All phthalates, polycyclic aromatic hydrocarbons (PAHs), and phenols were measured in urine. All polybrominated diphenyl ethers (PBDEs) were measured in serum and adjusted for lipid level. All polyfluorochemicals (PFCs) were measured in serum. In the metals model, $\mathrm{Pb}$ was measured in whole blood, while other metals were measured in urine. In models of metal subset and other chemicals (PAHs and Triclosan) blood Cd and total $\mathrm{Hg}$ measurements were used instead of urine measurements because urine metal measurements were not available for participants with PAHs and Triclosan. All urine measurements were adjusted for creatinine to account for differences in kidney function.

Six chemical mixtures were tested for association with SA colonization: metals, phthalates, PAHs, PBDEs, PFCs, and phenols. Three chemical mixtures were tested for association with MRSA: metals, metal subset + PAHs, and metal subset + triclosan. Mixtures used in MRSA models were determined based on known antibiotic properties, and data overlap.

2.2.2. Outcomes-Nasal swabs for SA detection were collected from eligible participants during the 2001-2004 years of the survey only. Colonization by SA was detected using standard culture based methods. SA isolates were tested for methicillin resistance by disk diffusion. Swabs that tested negative for SA were considered negative for both MRSA and SA. Swabs that tested positive for SA and for methicillin resistance were considered positive for MRSA. Swabs that tested positive for SA but negative for methicillin resistance were considered positive for SA but negative for MRSA.

2.2.3. Covariates-In order to increase comparability between statistical models while trying to avoid over adjustment, we adjusted all models for a base set of covariates that included age, gender, education, income, race/ethnicity, and smoking. For models that combined data across the two survey waves (metals, phthalates, PAHs, metal subset + PAHs), we also adjusted for survey wave (2001-2002, 2003-2004).

All data on covariates were self-reported by participants during an in-person interview. Age was modeled continuously, and gender as male or female. Education was modeled categorically as follows: K-12 aged (6-19 years old), shigh school/GED, some college, $\geq$ Bachelor Degree. Family income was converted to a poverty income ratio based on the federal poverty threshold for the year of data collection, released annually by the Department of Health and Human Services. Income was then modeled continuously. Race/ ethnicity was categorized as non-Hispanic White, non-Hispanic Black, Hispanic, and other. 
Smoking status was defined into categories of never smoker, former smoker, and current smoker.

\subsection{Statistical analysis}

WQS regression was used to assess associations between mixtures of chemical exposures and nasal colonization by SA and MRSA. WQS is a statistical method designed to evaluate the joint association of multiple potentially correlated exposure variables on a health outcome, estimating the effect of all exposure variables as a single weighted index (Carrico et al., 2015). Each exposure variable is assigned a weight within the index that indicates its importance in the mixture. We examined 6 different classes of chemical mixtures in association with SA (Table 1). We examined associations of mixtures of metals, and metals combined with PAHs and triclosan, respectively, with MRSA colonization (Table 1). Each WQS model was adjusted for covariates. Frequency tables were calculated using the full data used across all WQS models in SAS v.9.4.

WQS models were run using the gWQS v.2.0.0 in R v.3.6.1. The gWQS package does not currently support the use of complex survey structure variables, thus the NHANES survey weights were not used. Instead, we adjusted for variables used to calculate survey weights, as per previous studies (Park et al., 2017; Power et al., 2013). Within the gWQS function, reported results were estimated by specifying the use of deciles for exposure weighting, $40 \%$ of the data used as the test set with the other $60 \%$ used as the validation set, 100 bootstrap repetitions, the random seed set to 2019 , and a binomial distribution used for the general linear model. Each model used the same outcome (either SA or MRSA) in the test set and validation set. As a sensitivity analysis, because little evidence exists to indicate which direction associations may be, two models were run for each mixture, one with betas in the weight estimation constrained to be positive and one with betas constrained to be negative. In each case the weights are estimated by pooling only the effects in the positive or negative direction as constrained. For all models, except PBDEs, reported results were estimated with the betas constrained to positive (negative for PBDEs) because models in the opposite direction either showed no effect, or did not converge. Individual chemical weights of $\geq 0.1$ were considered most important. The value of 0.1 was chosen for ease of comparison across models that included different numbers of chemicals. Additional sensitivity analysis examined all models stratified by age, unadjusted for confounders to allow all models to be comparable and converge.

\section{RESULTS}

\subsection{Study population characteristics}

The analytical sample included participants 6-85 years old based on data availability. Data included in the analytical sample came from NHANES survey years 2001-2002 and 2003$2004,30.4 \%$ and $69.6 \%$, respectively (Table 2). Approximately $73 \%$ of the analytical sample was below age 50 . The sample was slightly more female (53.6\%), included a higher proportion of non-Hispanic Whites relative to other race/ethnic categories (42.6\%), included a large sample of individuals with a high school diploma or less (71.7\%), including the $32.6 \%$ percent of participants age 6-17. Most participants were never smokers $(70.8 \%)$, with 
a household income below 200\% of the Federal Poverty Level (50.8\%). 29.2\% of the sample was positive for nasal SA colonization, including 1.2\% with MRSA. Geometric means for all 66 exposure analytes can be found in the supplement (ST1).

\subsection{Mixture associations with Staphylococcus aureus}

Mixtures of chemicals by each class including metals, phthalates, PAHs, PBDEs, PFCs, and phenols were tested for associations with SA colonization separately. Increased nasal SA colonization was statistically significantly associated with mixtures of metals $(\mathrm{OR}=1.11$, $95 \% \mathrm{CI}=1.02-1.20)$, phthalates $(\mathrm{OR}=1.09,95 \% \mathrm{CI}=1.04-1.14)$, and phenols $(\mathrm{OR}=1.08$, $95 \% \mathrm{CI}=1.01-1.15$ ), in adjusted models (Fig 1). Sensitivity analysis of unadjusted models, stratified by age, indicated that effects were strongest in those below the age of 20 and above the age of 49 for metals, while effects were strongest for phthalates in those below the age of 20 (ST2). The model of PAHs showed a slight but statistically insignificant positive association with SA, while models of PBDEs were very close to null, and PFCs appeared to have a negative association with SA, with a large and statistically insignificant confidence interval (Fig 1).

The most heavily weighted component in the metal WQS model was urine mercury (weight $=0.27$ ) (Fig 2a). Other components with weights $\geq 0.1$ were urine tungsten $($ weight $=0.17)$, urine thallium (weight $=0.11)$, blood $\mathrm{Pb}$ (weight $=0.10$ ), and urine uranium $($ weight $=0.10$ ). In the phthalate WQS model, mono-benzyl phthalate was most heavily weighted (weight $=$ 0.49 ), followed by mono-cyclohexyl phthalate (weight $=0.14$ ), and mono-isobutyl phthalate (weight $=0.14)($ Fig 2b). 2-5-Dichlorophenol was the most heavily weighted component of the phenol WQS model (weight $=0.59$ ), with bisphenol A also above 0.1 (weight $=0.18$ )

(Fig 2c).

\subsection{Mixture associations with methicillin resistant Staphylococcus aureus}

The adjusted WQS model of metal subset + PAHs shows a statistically significant associated with increased odds of nasal MRSA colonization ( $\mathrm{OR}=1.38,95 \% \mathrm{CI}=1.02-1.86)$ (Fig 3a). Adjusted models of metals, and metal subset + triclosan showed slight, statistically insignificant associations. The most heavily weighted components of the metal subset + PAH model were: blood Cd (weight $=0.30), 2$-phenanthrene $($ weight $=0.28), 1$-napthol (weight $=0.16)$, blood $\mathrm{Hg}($ weight $=0.12)$, and 2-napthol $($ weight $=0.10)($ Fig 3b $)$.

\section{DISCUSSION}

This analysis of NHANES data revealed previously unreported associations between different classes of chemical mixtures and nasal SA colonization. Metals, phthalates, and phenols mixtures showed statistically significant associations with increased odds of SA colonization, with $\mathrm{Hg}$, mono-benzyl phthalate, and 2-5-dichlorophenol weighing the most heavily in each mixture association, respectively. A mixture of metals and PAHs also showed a statistically significant association with nasal colonization by MRSA, with $\mathrm{Cd}$ and 2-phenanthrene most heavily weighted. These results suggest that the combined exposures of many environmental chemicals are associated with components of the human microbiome including potentially pathogenic species like SA and antibiotic resistant MRSA. While

Environ Res. Author manuscript; available in PMC 2021 November 01. 
several of the mixtures examined did not show statistically significant associations, future studies of exposure to these chemical mixtures (PAHs, PBDEs, PFCs) should be done to investigate their relationship with other components of the human microbiome.

\subsection{Metals}

The mixture of metals was associated with an $11 \%$ increase in odds of nasal SA colonization, and had a negligible association with MRSA. Mercury, tungsten, thallium, and lead were most heavily weighted within the mixture. Results are consistent with previous animal and epidemiologic findings that heavy metal exposure is associated with changes in microbial community structure.

Toxic metal exposures have been identified among the top ten global threats to public health. Heavy metals are pervasive in the environment as natural substances, with increased human exposure due to human activity, including industrial, agricultural, technological and medical applications (Tchounwou et al., 2012). While some metals serve as essential nutrients, many metals serve no biological function, and exposure can lead to a plethora of detrimental health outcomes, including immune and neurological dysfunction. Much like in humans, heavy metals are toxic to many bacteria, therefore exposure of bacterial communities to heavy metals can lead to changes in the community structure by reducing abundance of susceptible bacteria and selecting for bacteria that are more resistant to the toxic effects of metal. Recent studies of the effects of $\mathrm{Pb}$ on the gut microbiome in mice found that $\mathrm{Pb}$ exposure reduced gut microbial alpha diversity, shifting the relative abundance of Firmicutes and Bacteroidetes, the two most abundant bacterial phyla in the human gut microbiota (Gao et al., 2017; Xia et al., 2018b, 2018a). Studies of exposure Cd, As, and Hg have found similar results (Ba et al., 2017; Chang et al., 2019; Chi et al., 2017; Lu et al., 2014). Experimental animal studies have shown that metal exposure also leads to altered metabolic profiles that include increased stress responses such as DNA repair, cellular membrane biosynthesis, and multiple-antibiotic resistance, as well as reduced carbohydrate metabolism and short-chain fatty acid synthesis (Chi et al., 2017; Lu et al., 2014).

While the results of this analysis are supportive of previous epidemiologic evidence that metals are important predictors of microbial composition and SA colonization, they also differ with respect to which metals are most significant. Thus, results from this analysis emphasize the importance of chemical mixtures analyses in estimating toxicity in real world settings. In a previous analysis, we examined the association between blood $\mathrm{Pb}$ and blood Cd individually and SA colonization (Eggers et al., 2018), also using the same years of NHANES data. We found blood $\mathrm{Pb}$ to be associated with increased odds of MRSA and decreased odds of methicillin susceptible Staphylococcus aureus (MSSA) colonization, while blood Cd was associated with decreased odds of both MSSA and MRSA. Differences seen between those individual analyses and the mixture analyses shown here may be due to several factors. With many correlated metals accounted for in this analysis, that were not considered in the previous single metal analysis, this analysis may show true interactive effects of the chemical exposures, or the results of the previous analysis may have been confounded by the other metals. In this analysis of metal mixtures, the sample size was also smaller by approximately 15,000 due to the metal analytes used, thus the signal may be 
different in this much smaller portion of the population. One last consideration is that the previous analysis classified SA colonization into two exclusive groups, MSSA and MRSA, and in this analysis we pooled both into the SA outcome, which may account for some of the differences in association.

\subsection{Metal and PAH mixtures}

The metal subset + PAH mixture was associated with a $38 \%$ increase in odds of nasal MRSA colonization. Blood cadmium and 2-phenanthrene were weighted most heavily in the chemical mixture.

PAHs are a common byproduct of burning coal, oil, wood, and other materials, and can also be found in some medicines and pesticides. Mixtures of metals and PAHs are ubiquitous in the environment (Curran et al., 2000; Sprovieri et al., 2007). Findings that mixtures of metals and PAHs are associated with MRSA colonization are consistent with mechanistic understanding of both chemical classes and relationships with microbial diversity and antibiotic resistance. Both metals and PAHs have shown individual toxicity to bacteria (Luo et al., 2017; Tóth et al., 2016) as well as the ability to select for antibiotic resistance (Rishi et al., 2017; Wang et al., 2017). In mixtures of metals and PAHs, their co-toxicity has the potential for more-than-additive effects. PAHs can induce membrane damage that leads to increased permeability of metals into bacterial cells (Gauthier et al., 2014). Both PAHs and metals can also modulate cellular detoxification pathways of the other (Cytochrome P450 1A1 enzymes, and metallothionein) (Gauthier et al., 2014). In both scenarios, these interactions lead to increased cellular concentration of metals, which can increase metal's toxicity to susceptible bacteria, and on a bacterial-population scale, increase selective pressure for antibiotic resistance genes. This mechanism may contribute to our finding of elevated odds of MRSA colonization with combined exposure to metals and PAHs. Interestingly, PAHs on their own were not significantly associated with SA carriage, and metals on their own were not associated with increased odds of MRSA carriage, again emphasizing the importance of chemical mixtures analyses in epidemiologic studies of environmental exposures.

\subsection{Phthalates}

The phthalate mixture examined in this analysis was associated with a 9\% increase in odds of nasal SA carriage, with mono-benzyl phthalate weighted most heavily in the chemical mixture.

Phthalates are often used in plastic products to increase their flexibility and durability, and humans are often exposed to them through the diet. A previous study of diethyl phthalate exposure in rats showed an altered gut microbiome when exposed in adolescence (Hu et al., 2016). They found increased relative abundance of Bacteroidetes and decreased Firmicutes, compared with control rats, however, the genus Staphylococcus was not found to be significantly different. Few other studies of phthalates and the mammalian microbiome exist, however, in an analysis of NHANES 2001-2004, Geller et al., found that increased exposure to some phthalates was associated with increased odds of bacterial vaginosis, a dysbiosis of the vaginal microbiota (Geller et al., 2018). When our results are considered together with 
these studies, they suggest an effect of phthalates on the human microbiome, however more investigation is needed.

\subsection{Phenols}

The phenol mixture assessed here was associated with an $8 \%$ increase in odds of nasal SA carriage, and 2-5-Dichlorophenol was most heavily weighted in the mixture.

Phenols are a class of chemicals found in foods, plastic products, household cleaners and medical products. Exposure to phenols has been associated with infertility, cancer, and metabolic disorders (Konieczna et al., 2015). Developmental exposure to Bisphenol A (BPA) has also been shown to dysregulate immune function, causing a shift toward autoimmune response, and reducing the immune system's ability to fight infection (Xu et al., 2016). Specifically, BPA exposure limits neutrophil ability to kill SA, including MRSA (Balistrieri et al., 2018). While evidence of direct interaction between BPA and the mammalian microbiome is limited, one study in mice found that BPA altered the gut microbiome composition, including reduced bacterial diversity, and increased abundance of Proteobacteria, which is common in dysbiosis (Lai et al., 2016).

\subsection{Strengths and Limitations}

This analysis contributes previously unreported evidence of association between human exposure to environmental chemical mixtures and SA and MRSA colonization, as part of the human microbiome. By using publicly available NHANES data, we were able to assess a broad range of chemical exposures using a relatively large sample size.

Our analysis was limited however, in that we could not assess all chemical exposure in one mixture due to sampling procedure and lack of data overlap. Using multiple subsets of NHANES data with available exposure assessments led to an overall demographic distribution that is younger than the typical distribution in most NHANES analysis, and may not be representative of the United States population. As with all cross-sectional survey data, we are unable to include exposure timing in our analysis, thus our analysis cannot assess causality. Moreover, combining exposure measurements from different sample matrixes (blood, urine) that reflect different windows of exposure may also muddy the interpretation of our results. For instance, blood metal measurements can reflect exposure on the order of months (National Research Council (US) Committee on Measuring Lead in Critical Populations, 1993), while urine measurement can either reflect exposure in recent weeks (Poddalgoda et al., 2017), or can indicate chronic exposure through kidney burden (Tchounwou et al., 2012).

Assumptions of our analytical approach were that data were missing at random, that the dose-response was consistent across quantiles of exposure to each chemical, and that associations were linear. Given that little is known about the relationship between chemical exposure mixtures and the human microbiome, these assumptions may or may not be reasonable. Moreover, we were not able to account for survey weights in our analysis, but did adjust for demographic variables. 


\title{
5. CONCLUSION
}

Our results support that exposure to chemical mixtures is associated with SA colonization, including MRSA. Antibiotic resistance, is a growing public health priority, and environmental factors may play a part in altering associations. Moreover, examining coexposure to multiple chemicals is important to estimate associations more in line with the real world setting.

\section{Supplementary Material}

Refer to Web version on PubMed Central for supplementary material.

\section{ACKNOWLEDGEMENTS}

\begin{abstract}
We would like to thank all involved with the National Health and Nutrition Examination Survey for the use of their data.

FUNDING

S.E. was supported by the Eunice Kennedy Shriver National Institute of Child Health and Human Development (T32 HD049311). C.G. was supported by the National Institute of Environmental Health Sciences through the Mount Sinai Transdisciplinary Center on Early Environmental Exposures Biostatistics and Bioinformatics Facility Core (P30ES023515). M.A. was supported by the National Institute of Environmental Health Sciences through several mechanisms (P30ES023515, DP2ES025453, R01ES026033, U2CES030859). No funding agency had any role in the design of the study, or in the collection, analysis, and interpretation of data, or in writing the manuscript.
\end{abstract}

\section{LIST OF ABBREVIATIONS}

SA

MRSA

WQS

NHANES

PAHs

PBDEs

PFCs

MSSA

BPA
Staphylococcus aureus

methicillin resistant Staphylococcus aureus

weighted quantile sum

National Health and Nutrition Examination Survey

polycyclic aromatic hydrocarbons

polybrominated diphenyl ethers

polyfluorochemicals

methicillin susceptible Staphylococcus aureus

Bisphenol A

\section{REFERENCES}

Ba Q, Li M, Chen P, Huang C, Duan X, Lu L, Li J, Chu R, Xie D, Song H, Wu Y, Ying H, Jia X, Wang H, 2017 Sex-Dependent Effects of Cadmium Exposure in Early Life on Gut Microbiota and Fat Accumulation in Mice. Environ. Health Perspect 125, 437-446. 10.1289/EHP360 [PubMed: 27634282]

Balistrieri A, Hobohm L, Srivastava T, Meier A, Corriden R, 2018 Alterations in human neutrophil function caused by bisphenol A. Am. J. Physiol. Cell Physiol 315, C636-C642. 10.1152/ ajpcell.00242.2017 [PubMed: 30088793] 
Bisanz JE, Enos MK, Mwanga JR, Changalucha J, Burton JP, Gloor GB, Reid G, 2014 Randomized open-label pilot study of the influence of probiotics and the gut microbiome on toxic metal levels in Tanzanian pregnant women and school children. mBio 5, e01580-01514. 10.1128/mBio.01580-14 [PubMed: 25293764]

Bopp SK, Barouki R, Brack W, Dalla Costa S, Dorne J-LCM, Drakvik PE, Faust M, Karjalainen TK, Kephalopoulos S, van Klaveren J, Kolossa-Gehring M, Kortenkamp A, Lebret E, Lettieri T, Nørager S, Rüegg J, Tarazona JV, Trier X, van de Water B, van Gils J, Bergman A, 2018 Current EU research activities on combined exposure to multiple chemicals. Environ. Int 120, 544-562. 10.1016/j.envint.2018.07.037 [PubMed: 30170309]

Carlin Danielle J, Rider Cynthia V, Woychik Rick, Birnbaum Linda S., 2013 Unraveling the Health Effects of Environmental Mixtures: An NIEHS Priority. Environ. Health Perspect 121, a6-a8. 10.1289/ehp.1206182 [PubMed: 23409283]

Carrico C, Gennings C, Wheeler DC, Factor-Litvak P, 2015 Characterization of Weighted Quantile Sum Regression for Highly Correlated Data in a Risk Analysis Setting. J. Agric. Biol. Environ. Stat 20, 100-120. 10.1007/s13253-014-0180-3 [PubMed: 30505142]

Chang X, Li H, Feng J, Chen Y, Nie G, Zhang J, 2019 Effects of cadmium exposure on the composition and diversity of the intestinal microbial community of common carp (Cyprinus carpio L.). Ecotoxicol. Environ. Saf 171, 92-98. 10.1016/j.ecoenv.2018.12.066 [PubMed: 30597321]

Chi L, Bian X, Gao B, Tu P, Ru H, Lu K, 2017 The Effects of an Environmentally Relevant Level of Arsenic on the Gut Microbiome and Its Functional Metagenome. Toxicol. Sci 160, 193-204. 10.1093/toxsci/kfx174 [PubMed: 28973555]

Curran KJ, Irvine KN, Droppo IG, Murphy TP, 2000 Suspended Solids, Trace Metal and PAH Concentrations and Loadings from Coal Pile Runoff to Hamilton Harbour, Ontario. J. Gt. Lakes Res 26, 18-30. 10.1016/S0380-1330(00)70670-8

Eggers S, Safdar N, Malecki KM, 2018 Heavy metal exposure and nasal Staphylococcus aureus colonization: analysis of the National Health and Nutrition Examination Survey (NHANES). Environ. Health Glob. Access Sci. Source 17, 2 10.1186/s12940-017-0349-7

Eggers S, Safdar N, Sethi AK, Suen G, Peppard PE, Kates AE, Skarlupka JH, Kanarek M, Malecki KMC, 2019 Urinary lead concentration and composition of the adult gut microbiota in a crosssectional population-based sample. Environ. Int. 133, 105122 10.1016/j.envint.2019.105122 [PubMed: 31518933]

Gao B, Chi L, Mahbub R, Bian X, Tu P, Ru H, Lu K, 2017 Multi-Omics Reveals that Lead Exposure Disturbs Gut Microbiome Development, Key Metabolites, and Metabolic Pathways. Chem. Res. Toxicol 30, 996-1005. 10.1021/acs.chemrestox.6b00401 [PubMed: 28234468]

Gauthier PT, Norwood WP, Prepas EE, Pyle GG, 2014 Metal-PAH mixtures in the aquatic environment: a review of co-toxic mechanisms leading to more-than-additive outcomes. Aquat. Toxicol. Amst. Neth 154, 253-269. 10.1016/j.aquatox.2014.05.026

Geller RJ, Brotman RM, O'Brien KM, Fine DM, Zota AR, 2018 Phthalate exposure and odds of bacterial vaginosis among U.S. reproductive-aged women, NHANES 2001-2004. Reprod. Toxicol. Elmsford N 82, 1-9. 10.1016/j.reprotox.2018.09.001

Hu J, Raikhel V, Gopalakrishnan K, Fernandez-Hernandez H, Lambertini L, Manservisi F, Falcioni L, Bua L, Belpoggi F, L Teitelbaum S, Chen J, 2016 Effect of postnatal low-dose exposure to environmental chemicals on the gut microbiome in a rodent model. Microbiome 4, 26 10.1186/ s40168-016-0173-2 [PubMed: 27301250]

Iszatt N, Janssen S, Lenters V, Dahl C, Stigum H, Knight R, Mandal S, Peddada S, González A, Midtvedt T, Eggesbø M, 2019 Environmental toxicants in breast milk of Norwegian mothers and gut bacteria composition and metabolites in their infants at 1 month. Microbiome 7, 34 10.1186/ s40168-019-0645-2 [PubMed: 30813950]

Konieczna A, Rutkowska A, Rachoń D, 2015 Health risk of exposure to Bisphenol A (BPA). Rocz. Panstw. Zakl. Hig 66, 5-11. [PubMed: 25813067]

Lai K-P, Chung Y-T, Li R, Wan H-T, Wong CK-C, 2016 Bisphenol A alters gut microbiome: Comparative metagenomics analysis. Environ. Pollut. Barking Essex 1987 218, 923-930. 10.1016/ j.envpol.2016.08.039 
Lu K, Abo RP, Schlieper KA, Graffam ME, Levine S, Wishnok JS, Swenberg JA, Tannenbaum SR, Fox JG, 2014 Arsenic Exposure Perturbs the Gut Microbiome and Its Metabolic Profile in Mice: An Integrated Metagenomics and Metabolomics Analysis. Environ. Health Perspect 122, 284-291. 10.1289/ehp.1307429 [PubMed: 24413286]

Luo J, Hein C, Mücklich F, Solioz M, 2017 Killing of bacteria by copper, cadmium, and silver surfaces reveals relevant physicochemical parameters. Biointerphases 12, 020301 10.1116/1.4980127 [PubMed: 28407716]

National Research Council (US) Committee on Measuring Lead in Critical Populations, 1993 Methods for Assessing Exposure to Lead. National Academies Press (US).

Park SK, Zhao Z, Mukherjee B, 2017 Construction of environmental risk score beyond standard linear models using machine learning methods: application to metal mixtures, oxidative stress and cardiovascular disease in NHANES. Environ. Health 16 10.1186/s12940-017-0310-9

Poddalgoda D, Macey K, Assad H, Krishnan K, 2017 Development of biomonitoring equivalents for barium in urine and plasma for interpreting human biomonitoring data. Regul. Toxicol. Pharmacol 86, 303-311. 10.1016/j.yrtph.2017.03.022 [PubMed: 28359824]

Power MC, Webster TF, Baccarelli AA, Weisskopf MG, 2013 Cross-sectional association between polyfluoroalkyl chemicals and cognitive limitation in the National Health and Nutrition Examination Survey (NHANES). Neuroepidemiology 40, 125-132. 10.1159/000342310 [PubMed: 23095808]

Rishi P, Thakur R, Kaur UJ, Singh H, Bhasin KK, 2017 Potential of 2, 2'-dipyridyl diselane as an adjunct to antibiotics to manage cadmium-induced antibiotic resistance in Salmonella enterica serovar Typhi Ty2 strain. J. Microbiol. Seoul Korea 55, 737-744. 10.1007/s12275-017-7040-0

Sprovieri M, Feo ML, Prevedello L, Manta DS, Sammartino S, Tamburrino S, Marsella E, 2007 Heavy metals, polycyclic aromatic hydrocarbons and polychlorinated biphenyls in surface sediments of the Naples harbour (southern Italy). Chemosphere 67, 998-1009. 10.1016/ j.chemosphere.2006.10.055 [PubMed: 17157354]

Tchounwou PB, Yedjou CG, Patlolla AK, Sutton DJ, 2012 Heavy Metals Toxicity and the Environment. EXS 101, 133-164. 10.1007/978-3-7643-8340-4_6 [PubMed: 22945569]

Tóth B, Liktor-Busa E, Kúsz N, Szappanos Á, Mándi A, Kurtán T, Urbán E, Hohmann J, Chang F-R, Vasas A, 2016 Phenanthrenes from Juncus inflexus with Antimicrobial Activity against Methicillin-Resistant Staphylococcus aureus. J. Nat. Prod 79, 2814-2823. 10.1021/ acs.jnatprod.6b00581 [PubMed: 27808510]

Wang J, Wang J, Zhao Z, Chen J, Lu H, Liu G, Zhou J, Guan X, 2017 PAHs accelerate the propagation of antibiotic resistance genes in coastal water microbial community. Environ. Pollut. Barking Essex 1987 231, 1145-1152. 10.1016/j.envpol.2017.07.067

Xia J, Jin C, Pan Z, Sun L, Fu Z, Jin Y, 2018a Chronic exposure to low concentrations of lead induces metabolic disorder and dysbiosis of the gut microbiota in mice. Sci. Total Environ 631-632, 439448. 10.1016/j.scitotenv.2018.03.053

Xia J, Lu L, Jin C, Wang S, Zhou J, Ni Y, Fu Z, Jin Y, 2018b Effects of short term lead exposure on gut microbiota and hepatic metabolism in adult zebrafish. Comp. Biochem. Physiol. Toxicol.

Pharmacol. CBP 209, 1-8. 10.1016/j.cbpc.2018.03.007

Xu J, Huang G, Guo TL, 2016 Developmental Bisphenol A Exposure Modulates Immune-Related Diseases. Toxics 4 10.3390/toxics4040023

Zhai Q, Cen S, Jiang J, Zhao J, Zhang H, Chen W, 2019 Disturbance of trace element and gut microbiota profiles as indicators of autism spectrum disorder: A pilot study of Chinese children. Environ. Res 171, 501-509. 10.1016/j.envres.2019.01.060 [PubMed: 30743242]

Zipf G, Chiappa M, Porter KS, Ostchega Y, Lewis BG, Dostal J, 2013 National health and nutrition examination survey: plan and operations, 1999-2010. Vital Health Stat. Ser 1 Programs Collect. Proced 1-37. 


\section{HIGHLIGHTS}

- $\quad$ Mixtures of exposures were assessed in association with S. aureus colonization.

- Mixtures within metals, phthalates, and phenols were associated with $S$. aureus.

- One mixture was associated with methicillin resistant $S$. aureus colonization.

- Findings support analysis of chemical mixtures and components of the microbiome. 


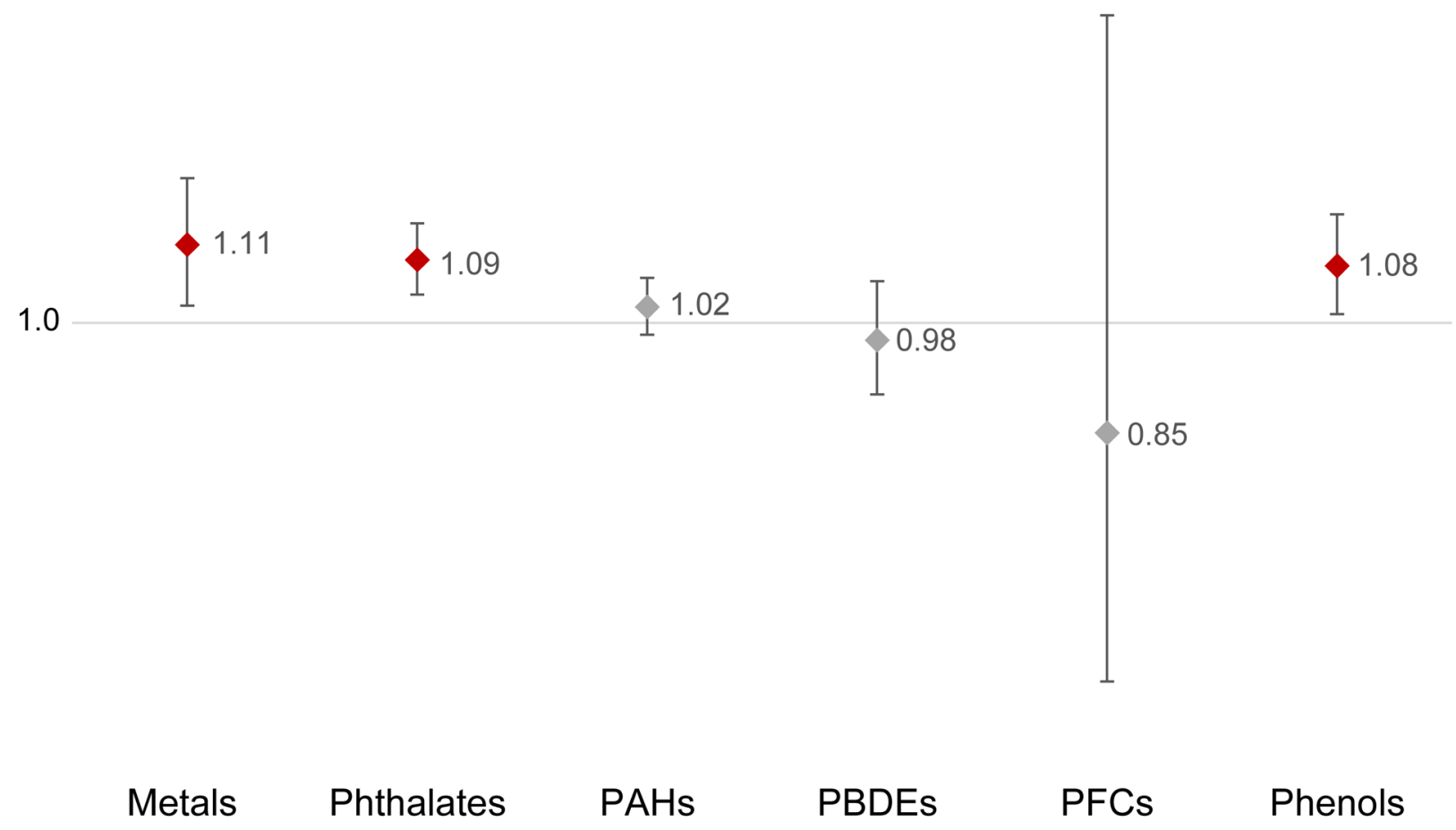

\section{Figure 1.}

Adjusted odds ratios of each WQS index on Staphylococcus aureus nasal colonization. Odds ratios with $95 \%$ confidence intervals that do not cross 1.00 are considered statistically significant, and indicated in red. Models were adjusted for age, education, gender, income, race/ethnicity, smoking status, and survey wave for models that included data from multiple waves (metals, phthalates, PAHs). 


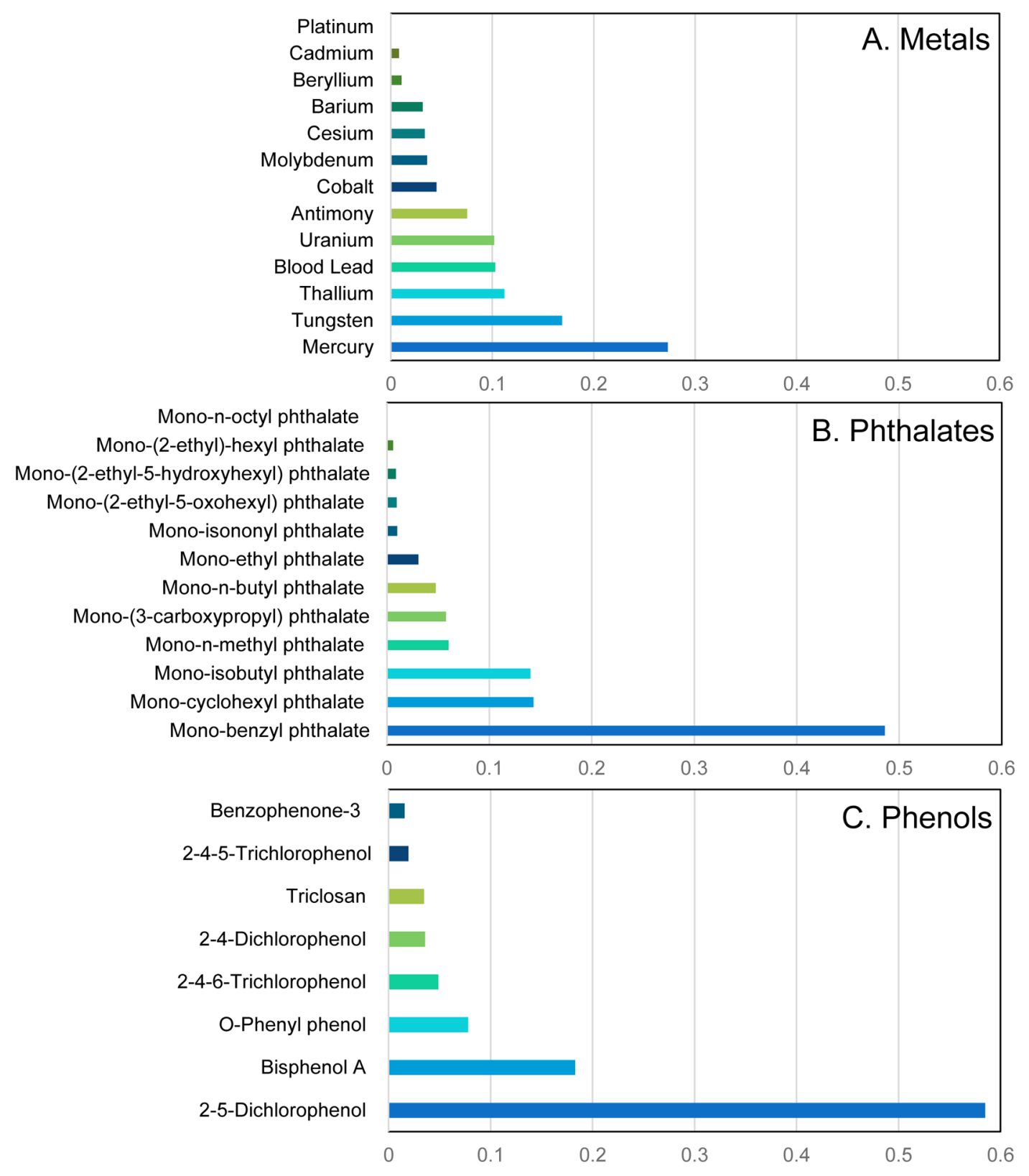

Figure 2.

WQS index weights for the indexes that were statistically significantly associated with Staphylococcus aureus nasal colonization: A. Metal index, B. Phthalate index, C. Phenol index. 
A. Odds Ratios of MRSA

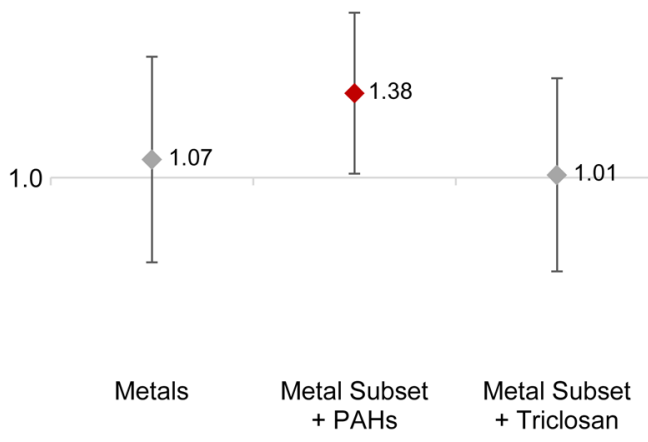

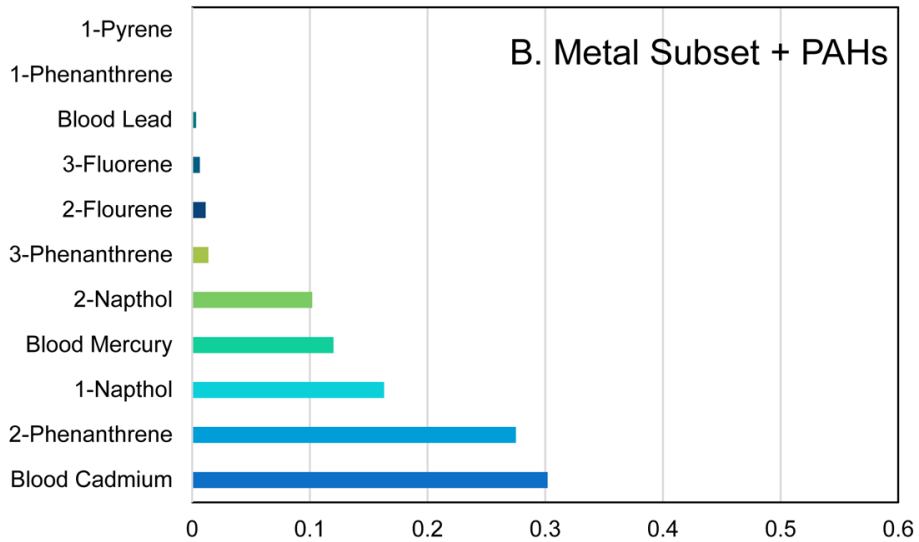

Figure 3.

A. Adjusted odds ratios of each WQS index on Methicillin Resistant Staphylococcus aureus nasal colonization. Odds ratios with $95 \%$ confidence intervals that do not cross 1.00 are considered statistically significant, and indicated in red. Models were adjusted for age, education, gender, income, race/ethnicity, smoking status, and survey wave for the model that included data from multiple waves (metals). 
Table 1.

Details of 66 chemicals included in the weighted quantile sum (WQS) regression models. Sample size, years of data collection, and outcome tested with each index are indicated.

\begin{tabular}{|c|c|}
\hline WQS Chemical Index & Chemical Exposures \\
\hline $\begin{array}{l}\text { Metals }(\mathbf{1 3}) \\
\mathrm{n}=2825 \\
\text { Years: } 2001-2002,2003-2004 \\
\text { Outcome: SA, MRSA }\end{array}$ & $\begin{array}{l}\text { Blood Lead } \bullet \text { Urine Barium } \bullet \text { Urine Beryllium } \bullet \text { Urine Cadmium } \bullet \text { Urine Cobalt } \bullet \text { Urine Cesium } \bullet \text { Urine } \\
\text { Molybdenum } \bullet \text { Urine Platinum } \bullet \text { Urine Antimony } \bullet \text { Urine Thallium } \bullet \text { Urine Tungsten } \bullet \text { Urine Uranium } \bullet \text { Urine } \\
\text { Mercury }\end{array}$ \\
\hline $\begin{array}{l}\text { Phthalates (12) } \\
\mathrm{n}=4998 \\
\text { Years: } 2001-2002,2003-2004 \\
\text { Outcome: SA }\end{array}$ & $\begin{array}{l}\text { Mono-n-butyl phthalate } \bullet \text { Mono-(3-carboxypropyl) phthalate } \bullet \text { Mono-cyclohexyl phthalate } \bullet \text { Mono-ethyl } \\
\text { phthalate } \bullet \text { Mono-(2-ethyl-5-hydroxyhexyl) phthalate } \bullet \text { Mono-(2-ethyl)-hexyl phthalate } \bullet \text { Mono-isobutyl } \\
\text { phthalate } \bullet \text { Mono-n-methyl phthalate } \bullet \text { Mono-isononyl phthalate } \bullet \text { Mono-(2-ethyl-5-oxohexyl) phthalate } \\
\text { Mono-n-octyl phthalate } \bullet \text { Mono-benzyl phthalate }\end{array}$ \\
\hline $\begin{array}{l}\text { PAHs }(\mathbf{8}) \\
\mathrm{n}=4677 \\
\text { Years: } 2001-2002,2003-2004 \\
\text { Outcome: SA }\end{array}$ & $\begin{array}{l}\text { 1-Napthol } \bullet \text { 2-Napthol } \bullet \text {-Fluorene } \bullet \text { 2-Fluorene } \bullet \text { 3-Phenanthrene } \bullet \text { 1-Phenanthrene } \bullet \text { 2-Phenanthrene } \bullet \text { - } \\
\text { Pyrene }\end{array}$ \\
\hline $\begin{array}{l}\text { PBDEs }(11) \\
\mathrm{n}=1756 \\
2003-2004 \\
\text { Outcome: SA }\end{array}$ & 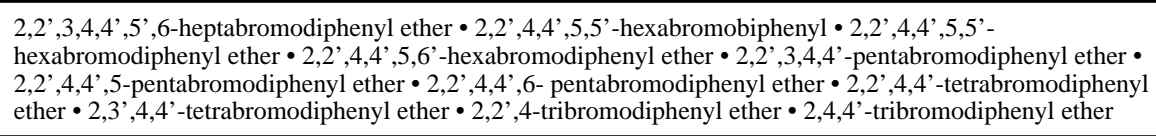 \\
\hline $\begin{array}{l}\text { PFCs (12) } \\
\mathrm{n}=1987 \\
\text { Years: } 2003-2004 \\
\text { Outcome: SA }\end{array}$ & $\begin{array}{l}\text { 2-(N-ethyl-PFOSA) acetate } \bullet \text { 2-(N-methyl-PFOSA) acetate } \bullet \text { Perfluorobutane sulfonic acid } \bullet \\
\text { Perfluorodecanoic acid } \bullet \text { Perfluorododecanoic acid } \bullet \text { Perfluoroheptanoic acid } \bullet \text { Perfluorohexane sulfonic acid } \\
\bullet \text { Perfluorononanoic acid } \bullet \text { Perfluorooctanoic acid } \bullet \text { Perfluorooctane sulfonic acid } \bullet \text { Perfluorooctane } \\
\text { sulfonamide } \bullet \text { Perfluoroundecanoic acid }\end{array}$ \\
\hline $\begin{array}{l}\text { Phenols }(8) \\
\mathrm{n}=2366 \\
\text { Years: } 2003-2004 \\
\text { Outcome: SA }\end{array}$ & $\begin{array}{l}\text { Bisphenol A } \bullet \text { Benzophenone-3 } \cdot \text { Triclosan } \bullet 2-5 \text {-Dichlorophenol } \bullet \text { 2-4- Dichlorophenol } \bullet \text { 2-4-5- } \\
\text { Trichlorophenol } \bullet 2-4-6 \text {-Trichlorophenol } \bullet \text { O- Phenyl phenol }\end{array}$ \\
\hline $\begin{array}{l}\text { Metal Subset + PAHs (11) } \\
\mathrm{n}=2623 \\
\text { Years: } 2001-2002,2003-2004 \\
\text { Outcome: MRSA }\end{array}$ & $\begin{array}{l}\text { 1-Napthol } \bullet \text { 2-Napthol } \bullet \text { 3-Fluorene } \bullet \text { 2-Fluorene } \bullet \text { 3-Phenanthrene } \bullet \text { 1-Phenanthrene } \bullet \text { 2-Phenanthrene } \bullet \text { 1- } \\
\text { Pyrene } \bullet \text { Blood Lead } \bullet \text { Blood Mercury } \bullet \text { Blood Cadmium }\end{array}$ \\
\hline $\begin{array}{l}\text { Metal Subset + Triclosan (4) } \\
\text { n=2277 } \\
\text { Years: } 2003-2004 \\
\text { Outcome: MRSA }\end{array}$ & Blood Lead $\bullet$ Blood Mercury $\bullet$ Blood Cadmium $\bullet$ Urine Triclosan \\
\hline
\end{tabular}


Table 2.

Demographics among participants across all models. NHANES 2001-2004.

\begin{tabular}{|c|c|c|}
\hline & $\mathbf{n}$ & $\%$ \\
\hline Total & 10312 & 100.0 \\
\hline \multicolumn{3}{|l|}{ Age } \\
\hline $6-17$ & 3358 & 32.6 \\
\hline $18-29$ & 1963 & 19.0 \\
\hline $30-49$ & 2233 & 21.7 \\
\hline $50-69$ & 1606 & 15.6 \\
\hline $70+$ & 1152 & 11.2 \\
\hline \multicolumn{3}{|l|}{ Gender } \\
\hline Female & 5526 & 53.6 \\
\hline Male & 4786 & 46.4 \\
\hline \multicolumn{3}{|l|}{ Education } \\
\hline K-12 Aged (6-19 years) & 4085 & 39.6 \\
\hline$\leq$ High School/GED & 3307 & 32.1 \\
\hline Some College & 1712 & 16.6 \\
\hline$\geq$ Bachelor Degree & 1208 & 11.7 \\
\hline \multicolumn{3}{|l|}{ Race/Ethnicity } \\
\hline Non-Hispanic White & 4396 & 42.6 \\
\hline Non-Hispanic Black & 2616 & 25.4 \\
\hline Hispanic & 2899 & 28.1 \\
\hline Non-Hispanic Other & 401 & 3.9 \\
\hline \multicolumn{3}{|l|}{ Income } \\
\hline$<200 \%$ FPL & 5236 & 50.8 \\
\hline 200-399\% FPL & 2742 & 26.6 \\
\hline$\geq 400 \%$ FPL & 2334 & 22.6 \\
\hline \multicolumn{3}{|l|}{ Smoking } \\
\hline Never Smoker & 7304 & 70.8 \\
\hline Former Smoker & 1604 & 15.6 \\
\hline Current Smoker & 1404 & 13.6 \\
\hline \multicolumn{3}{|l|}{ Survey Year } \\
\hline 2001-2002 & 3132 & 30.4 \\
\hline 2003-2004 & 7180 & 69.6 \\
\hline \multicolumn{3}{|l|}{ Staphylococcus aureus } \\
\hline Positive & 3014 & 29.2 \\
\hline Methicillin Resistant & 125 & 1.2 \\
\hline
\end{tabular}

Environ Res. Author manuscript; available in PMC 2021 November 01. 